\title{
GAUGE-POTENTIAL APPROACH TO THE KINEMATICS OF A MOVING CAR
}

\author{
Marián Fecko ${ }^{a}$ ) \\ Department of Theoretical Physics, Comenius University \\ Mlynská dolina F2, 84215 Bratislava, Slovakia
}

\begin{abstract}
A kinematics of the motion of a car is reformulated in terms of the theory of gauge potentials. $\mathrm{E}(2)$-gauge structure originates in the no-slipping contact of the car with a road.
\end{abstract}

\section{Introduction}

The physically most important field where the mathematical theory of connections ( $\leftrightarrow$ gauge potentials $\leftrightarrow$ Yang-Mills potentials) is used with great success is undoubtedly the theory of elementary particles. Since, however, the concepts involved are rather abstract and (especially for a newcomer in the field) mixed with a number of other (equally abstract) ones, one should appreciate to find out that gauge potentials can be used in 'much more mundane, but in return more readily visualized, context' [1], too, viz. in the context of classical mechanics [1],[3],[4],[5] or hydrodynamics [2]. A nice example of this sort is given in [1] (cf.also [3]). It was shown there that the natural kinematical framework for computing the net rotation of a (deformable) body due to a sequence of deformations is the non-Abelian gauge structure over the space of shapes of the body.

In this paper we show that (and rather in detail how) the kinematics of a motion of a car on a road can be reformulated in terms of non-Abelian gauge potentials, too. The gauge group is $E(2)$, the Euclidean group of the translations and rotations of the 2-dimensional plane.

It should be noted that the differential geometric treatment of the car's kinematics was given before in [6]. The new point here is the addition of the degree of freedom $\alpha$ (see Sec.2) which makes it possible then to treat the problem in the language of connections.

Finally let us mention a technical simplification made in computations. As is well known, the front (as well as rear) wheels of a car do not rotate with the same angular velocity in general (the device called differential is needed). When we speak about the angle $\alpha$ as being the angle measuring the orientation of the front wheel, the average angle is understood in fact. Or, equivalently, we compute everything as if the car was a tricycle (then $\alpha$ is the angle of the front wheel). The full account of the situation with two wheels can be done, of course, but it does not bring anything conceptually new. 


\section{The configuration space of a car as a principal $\mathrm{E}(2)$-bundle}

Let $P$ be the configuration space of a car. The coordinates $(\alpha, \beta, x, y, \varphi)$ are introduced according to the Fig.1, Fig.2, with the following meaning : $(x, y)$ are the Cartesian coordinates of the center of the front axle, $\varphi$ is the angle between the $x_{1}$ axis and the tie rod ('if that is the name of the thing connecting the front and rear axles' [6]; it measures the direction in which the car is headed), $\alpha$ measures the orientation of the front wheel with respect to the axle and $\beta$ is the angle made by the front axle with the tie rod. Thus $(x, y, \varphi)$ carry the information about the position of the tie rod alone in the $x_{1} x_{2}$ - plane irrespective of the 'shape' of the car whereas $(\alpha, \beta)$ encode the car's shape regardless of the position of the tie rod in the $x_{1} x_{2}$ - plane.

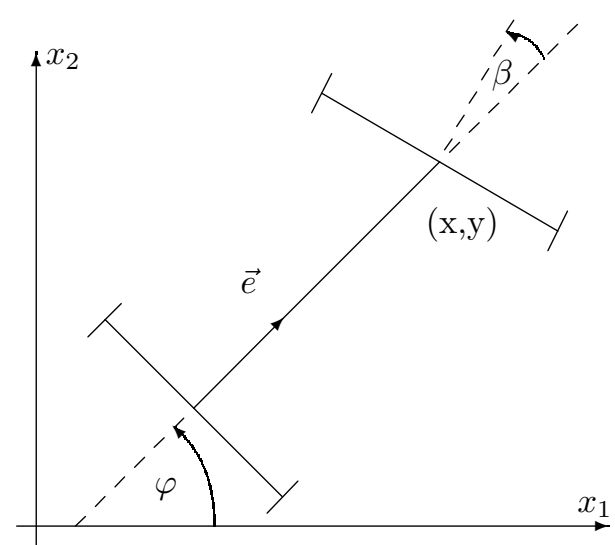

Fig.1 : The coordinates $x, y, \varphi, \beta$.

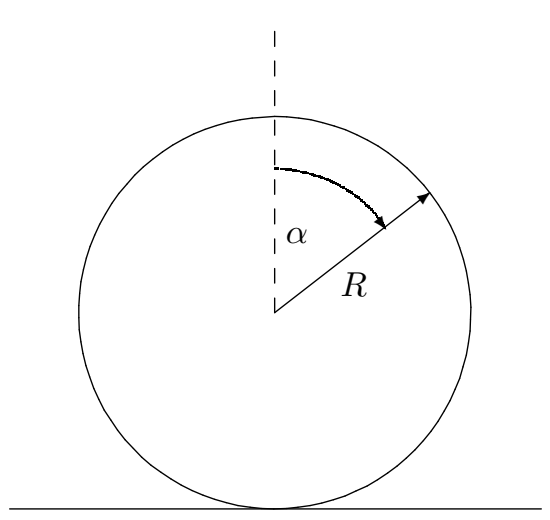

Fig.2 : The front wheel - the coordinate $\alpha$.

There is a natural action of the Euclidean group $E(2)$ on $P$, consisting in 'rigid' motions (rotations and translations) of the car with no change of its shape, that is to say the motions of the tie rod keeping the shape fixed. This action $R_{\mathcal{B}}: P \rightarrow P$ (see Appendix A for more technical details) results in the additional structure of the space $P$, viz. the structure of a principal fiber bundle with the group $E(2)$. It is constructed as follows : two configurations $p, p^{\prime} \in P$ are declared to be equivalent if they differ only by a rigid motion from $E(2)$, i.e. if there exists such $(B, b) \in E(2)$ that the action of $(B, b)$ on $p$ results in $p^{\prime}$, i.e. $R_{\mathcal{B}} p=p^{\prime}$. We define then $M$ as the factor-space $P / E(2)$, i.e. the points of $M$ are by definition the equivalence classes in $P$. There is a projection map

$$
\pi: P \rightarrow M
$$

sending the configuration $p$ to its own equivalence class $[p] \equiv \pi(p)=m$, or in coordinates

$$
\pi:(\alpha, \beta, x, y, \varphi) \mapsto(\alpha, \beta)
$$

Thus $\pi$ extracts from the complete configuration the information about the shape of the car and 'forgets' the position of the tie rod within the $x_{1} x_{2}$ - plane.

According to the terminology of [1],[2], $P$ is the space of 'located shapes' whereas $M$ is the space of 'unlocated shapes'.

If $m \in M$, the set $\pi^{-1}(m) \subset P$ (all those $p \in P$ which project to the fixed $m \in M$ ) is called the fiber over $m$ and here it represents all configurations (三 'located shapes') sharing the same ('unlocated') shape. Any two fibers $\pi^{-1}(m), \pi^{-1}\left(m^{\prime}\right)$ are mutually diffeomorphic (equally looking) and their abstract model, the typical fiber, is denoted by $\mathcal{E}$ (the space of the locations of the tie rod) in Appendix A and happens to be diffeomorphic to the group $E(2)$ itself.

Notice that the knowledge of the configuration $p \in P$ is equivalent (globally) to the knowledge of the ordered pair $(m, e) \in M \times \mathcal{E}$. In other words our total space $P$ of the bundle is (diffeomorphic to) the product $M \times \mathcal{E}$ of the base $M$ and the typical fiber $\mathcal{E}$

$$
P=M \times \mathcal{E}
$$


and the bundle projection $\pi$ is realized as a projection $\pi_{1}$ on the first factor

$$
\pi_{1}: M \times \mathcal{E} \rightarrow M \quad(m, e) \mapsto m
$$

This means that our bundle is trivial (in general this is the case only locally).

The section of the bundle $\pi: P \rightarrow M$ (the fixation of the gauge) is a map

$$
\sigma: M \rightarrow P
$$

obeying

$$
\pi \circ \sigma=\text { identity on } M
$$

$(\sigma(m)$ is to be in the fiber over $m)$. It helps to visualize the abstract shapes (elements of $M$ ) localizing each of them somewhere in the $x_{1} x_{2}$ - plane. The convenient (global) section is given in coordinates by

$$
\sigma:(\alpha, \beta) \mapsto(\alpha, \beta, 0,0,0)
$$

It realizes all shapes by means of the configurations with the tie rod situated at the $x_{1}$-axis to the left with respect to the origin (Fig.3). Notice that the coordinates $(x, y, \varphi)$ are closely related (adapted) to this very section (in fact they are introduced just with respect to this section) : the section defines (for all $m \in M$ ) the fiducial point $\sigma(m)$ in the fiber over $m$. This point is (by definition) labeled by the coordinates $(\alpha, \beta, 0,0,0)$. Then a general point $p$ in the same fiber (with the same shape) acquires the coordinates $(\alpha, \beta, x, y, \varphi)$ if the element $(B, b) \in E(2)$ with

$$
B=\left(\begin{array}{cc}
\cos \varphi & \sin \varphi \\
-\sin \varphi & \cos \varphi
\end{array}\right) \quad b=(x, y)
$$

is needed to obtain $p$ from $\sigma(m)$ via the group action.

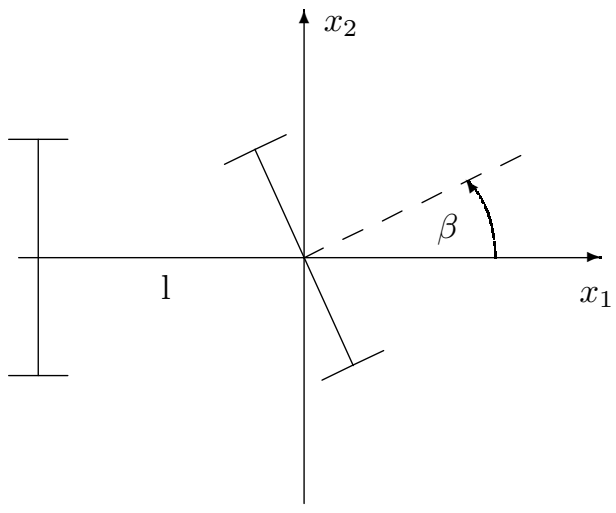

Fig.3 : The gauge fixation $\sigma$.

The useful possibility is to interpret the section (2.1) as the point of view of the driver (the driver's reference system) : with respect to his axes $x^{\prime}{ }_{1}, x^{\prime}{ }_{2}$ the tie rod is clearly always at the origin and directed forward $(x=y=\varphi=0)$. Each other choice of a section (other gauge) corresponds to some different observer, which can, however, depend on the (unlocated) shape.

\section{The no-slipping contact with a road as a connection on $\pi: P \rightarrow M$}

So far we have come to conclusion that the 5-dimensional configuration space $P$ of a car can be treated naturally as a total space of a (trivial) principal $\mathrm{E}(2)$-bundle $\pi: P \rightarrow M, P \equiv M \times \mathcal{E}$. A motion of the car on a road $\left(x_{1} x_{2}\right.$-plane) is given by a curve $\gamma(t) \equiv(m(t), e(t))$ on $P \equiv M \times \mathcal{E}$. The essential point is, however, that it is only the projection $m(t) \equiv \pi \circ \gamma(t)$ which is under direct control of a driver $(\alpha(t)$ - gas pedal, braces; $\beta(t)$ 
- steering wheel). The driver governs directly the 'motion' in the space of shapes $M$ (the base of the bundle) whereas what is really his goal is the change of the position of the tie rod, or in other words to move along the desired curve $e(t)$ in the typical fiber $\mathcal{E}$ of the bundle. The necessary 'bridge' between $M$ and $\mathcal{E}$ is given by a system of (anholonomic) differential constraints representing physically the condition of the no-slipping contact of the wheels with the road. In such a way the driver's activity represented as the curve $m(t)$ on $M$ is transformed to the curve $e(t)$ on $\mathcal{E}$ or, equivalently, $\gamma(t) \equiv(m(t), e(t))$ on $P$. As we will see, the procedure of the reconstruction of the complete $\gamma(t)$ on $P$ from its projection $m(t)$ on $M$ is just the horizontal lift $m \mapsto m^{h} \equiv \gamma$, where the structure necessary for it, viz. the connection in the principal bundle $\pi: P \rightarrow M$ (gauge structure over $\mathrm{M}$ ) enters the scene as a mathematical expression of the above mentioned no-slipping contact of the car with the road, i.e. the constraints of contact can be interpreted in terms of the connection form on $P$.

In general a connection on a principal fiber bundle $\pi: P \rightarrow M$ with a group $G$ is given [7] by a $\mathcal{G}$-valued $(\mathcal{G}$ being the Lie algebra of the group $G$ ) 1 -form on $P$, a connection form. In our case it means the $3 \times 3$ matrix of 1-forms on $P$ decomposable with respect to the basis $e_{0}, e_{1}, e_{2}$ of the Lie algebra $e(2)$ of the group $E(2)$ (see Appendix B)

$$
\begin{gathered}
\omega=\omega^{a} e_{a}=\omega^{0} e_{0}+\omega^{1} e_{1}+\omega^{2} e_{2}= \\
=\left(\begin{array}{ccc}
0 & \omega^{0} & 0 \\
-\omega^{0} & 0 & 0 \\
\omega^{1} & \omega^{2} & 0
\end{array}\right)
\end{gathered}
$$

where $\omega^{0}, \omega^{1}, \omega^{2}$ are 1 -forms on $P$. Thus the condition of the horizontality

$$
\omega=0 \quad \text { i.e. } \quad \omega^{0}=\omega^{1}=\omega^{2}=0
$$

represents just 3 independent relations between the differentials $d \alpha, d \beta, d x, d y, d \varphi$ enabling one to express the infinitesimal changes $\delta x, \delta y, \delta \varphi$ of the coordinates of the rod in terms of the given changes $\delta \alpha, \delta \beta$ of the coordinates of the shape of the car.

Note : the equations $\omega^{a}=0$ are not to be interpreted as 1-form identities on $P$ but rather in the sense that the forms are annihilated (give zero) by the velocity (三 tangent) vectors to the real (三 obeying the constraints $\Rightarrow$ by definition horizontal) trajectories on $P$.

The computation of the explicit expression for the connection form is performed in Appendix C. The result reads

$$
\begin{gathered}
\omega^{0}=d \varphi-\frac{R}{l} \sin \beta d \alpha \\
\omega^{1}=d x+y \omega^{0}-R \cos (\beta+\varphi) d \alpha \\
\omega^{2}=d y-x \omega^{0}-R \sin (\beta+\varphi) d \alpha
\end{gathered}
$$

If one fixes the gauge by choosing the section $\sigma$ (Sec.2), the gauge potential (in gauge $\sigma$ ) is given as

$$
\begin{gathered}
\mathcal{A}:=\sigma^{*} \omega=\mathcal{A}^{a} e_{a}=\mathcal{A}^{0} e_{0}+\mathcal{A}^{1} e_{1}+\mathcal{A}^{2} e_{2}=\left(\begin{array}{ccc}
0 & \mathcal{A}^{0} & 0 \\
-\mathcal{A}^{0} & 0 & 0 \\
\mathcal{A}^{1} & \mathcal{A}^{2} & 0
\end{array}\right)= \\
=-\frac{R}{l}\left(\begin{array}{ccc}
0 & \sin \beta & 0 \\
-\sin \beta & 0 & 0 \\
l \cos \beta & l \sin \beta & 0
\end{array}\right) d \alpha
\end{gathered}
$$

\section{Reconstruction of $\gamma(t)$ on $P$ from $\pi(\gamma(t))$ on $M$ as a horizontal lift}

The driver's activity is represented by a curve $m(t) \equiv \pi(\gamma(t))$ on $M$ (a sequence of shapes parametrized by time). The contact of the wheels with the road results then in a motion in the total configuration space $P$. According to the meaning of the connection as an object encoding all constraints of the contact, the resulting trajectory $\gamma(t)$ on $P$ is the horizontal lift of the curve $m(t)$, i.e. the unique curve $m^{h}(t)$ on $P$ enjoying the following two properties : 
i) $\pi\left(m^{h}(t)\right)=m(t) \quad \leftrightarrow \quad m^{h}(t)$ is always exactly 'over' $m(t)$

ii) $\dot{m}^{h} \equiv$ its tangent (velocity) vector - is always horizontal, i.e. it annihilates $\omega^{a}, a=0,1,2$.

Let us express these conditions in coordinates. If

$$
m(t) \leftrightarrow(\alpha(t), \beta(t))
$$

is given, then its horizontal lift is

$$
m^{h}(t) \leftrightarrow(\alpha(t), \beta(t), x(t), y(t), \varphi(t))
$$

(the same $\alpha$ and $\beta$ are there because of the condition $i) ; x, y, \varphi$ are to be determined). Now

$$
\dot{m}^{h}(t)=\dot{\alpha}(t) \partial_{\alpha}+\dot{\beta}(t) \partial_{\beta}+\dot{x}(t) \partial_{x}+\dot{y}(t) \partial_{y}+\dot{\varphi}(t) \partial_{\varphi}
$$

and

$$
<\omega^{a}, \dot{m}^{h}(t)>=0 \quad a=0,1,2
$$

gives

$$
\begin{gathered}
\dot{\varphi}=\dot{\alpha} \frac{R}{l} \sin \beta \\
\dot{x}=\dot{\alpha} R \cos (\beta+\varphi) \\
\dot{y}=\dot{\alpha} R \sin (\beta+\varphi)
\end{gathered}
$$

so that

$$
\dot{m}^{h}=\dot{\alpha}(t) H_{\alpha}+\dot{\beta}(t) H_{\beta}
$$

where

$$
\begin{gathered}
H_{\alpha} \equiv \partial_{\alpha}^{h}:=\partial_{\alpha}+R \cos (\beta+\varphi) \partial_{x}+R \sin (\beta+\varphi) \partial_{y}+\frac{R}{l} \sin \beta \partial_{\varphi} \\
H_{\beta} \equiv \partial_{\beta}^{h}:=\partial_{\beta}
\end{gathered}
$$

are the horizontal lifts of the coordinate basis vectors on $M$.

The 1-st order linear autonomous system of equations (4.1), the parallel transport equations, solves the reconstruction problem : given $\alpha(t), \beta(t)$ for $t \in<t_{i}, t_{f}>$ (sequence of shapes) and $\left(x\left(t_{i}\right), y\left(t_{i}\right), \varphi\left(t_{i}\right)\right.$ (the initial position in the fibre over $m\left(t_{i}\right)$, i.e. the initial position of the car on the road), it provides the remaining information about the motion of the car, viz. the sequence of the positions of the tie rod corresponding to the given sequence of shapes (an example - the motion with the fixed steering wheel - is computed in Appendix D). The parallely transported configuration is then by definition the configuration $m^{h}\left(t_{f}\right)$. (Recall that according to the meaning of the connection here to follow the parallel transport rule is the same thing as to be compatible with the constraints of the contact).

Note that the equations (4.1) are invariant (as is the case in general for the parallel transport equations) with respect to reparametrization - the speed of the shape sequence is irrelevant, what matters is only the path corresponding to $m(t)$ rather then the curve $m(t)$ itself. Surprisingly, this rather subtle technical fact seems to be pretty well known intuitively to our wives when they prevent us to drive too quickly ('you win nothing by it').

\section{Parking cycles as a clever use of the curvature $\Omega$ of the connection}

To get out of an extremely tight parking spot [6] a pure translation of the tie rod perpendicular to the latter, i.e. (infinitesimally)

$$
(x, y, \varphi) \mapsto(x-\epsilon \sin \varphi, y+\epsilon \cos \varphi, \varphi)
$$

$(\epsilon \ll 1)$ is strongly desirable lest we come to contact with the neighbouring car (and even much worse with the owner of the neighbouring car, then).

On the other hand according to the results of Sec.4. only the motions generated by some horizontal lift $\dot{m}^{h}$ are possible (allowed by the constraints), i.e. (cf. (4.2) - (4.4))

$$
(x, y, \varphi) \mapsto\left(x+\epsilon \cos (\varphi+\beta), y+\epsilon \sin (\varphi+\beta), \varphi+\frac{\epsilon}{l} \sin \beta\right)
$$


$(\epsilon=\dot{\alpha} R \delta t \ll 1)$. In no special case (5.2) reduces to (5.1) : (5.2) consists of both translation and rotation except for the case $\beta=0$, when, however, the translation is just along the tie rod. Thus it seems that we are simply unlucky and we have to wait until the car in front of us leaves.

This conclusion is, however, too hasty, since we have not used yet the basic parking algorithm known to every driver, viz. a cycle in the space $M$. Let us study for a moment the result of an infinitesimal cycle of the following structure (see Fig.4) :

i) go forth $(\alpha \mapsto \alpha+\epsilon)$

ii) turn the steering wheel to the left $(\beta \mapsto \beta+\epsilon)$

iii) go (the same step) back $(\alpha+\epsilon \mapsto \alpha)$

iv) turn the steering wheel (the same angle) back to the right $(\beta+\epsilon \mapsto \beta)$

Clearly we finish at (exactly) the same point in $M$; the complete configuration $p \equiv(m, e)$, however, changes : $p \equiv(m, e) \mapsto\left(m, e^{\prime}\right) \equiv p^{\prime}$, viz. up to the second order terms in $\epsilon$ the result is (see below)

$$
(\alpha, \beta, x, y, \varphi) \mapsto\left(\alpha, \beta, x-\epsilon^{2} R \sin (\varphi+\beta), y+\epsilon^{2} R \cos (\varphi+\beta), \varphi+\epsilon^{2} \frac{R}{l} \cos \beta\right)
$$

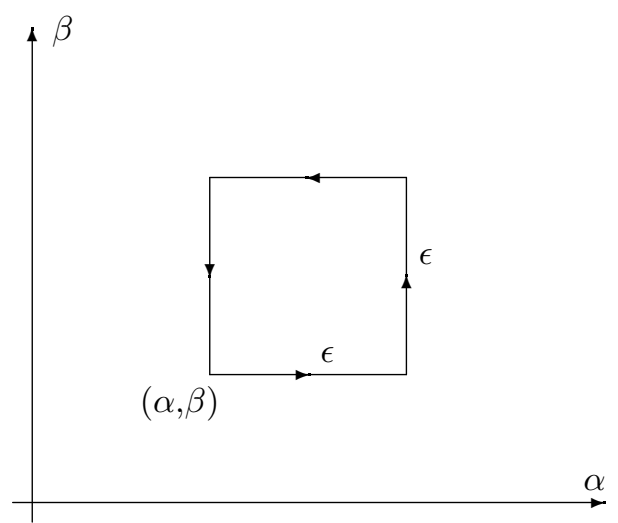

Fig.4 : A simple ifinitesimal parking cycle.

Although this does not meet our requirements yet (rotation is present unless $\beta=\frac{\pi}{2}$; if $\beta$ is $\frac{\pi}{2}$, the translation is once more along the tie rod) there is still something interesting here which will turn out to be the essential clue for the real solution of the parking problem. Namely, if one interprets (5.3) as a direct step from $p$ to $p^{\prime}$ (and not as the effective one $\equiv$ the result of the cyclic motion described above), it is forbidden (it violates the constraints). This particular step is even forbidden in a 'maximal way' - it is purely vertical (projects to the same point in $M$; both $p$ and $p^{\prime}$ lie in the same fibre). Thus the fact of vital importance is that a cycle composed exclusively of allowed ( $\equiv$ horizontal) steps can result in the (directly) forbidden motion (三 non-vanishing vertical part). This means that although we have come to the conclusion that (5.1) cannot be realized 'directly' (as one step) there is still a real hope to produce it effectively - as a result of (maybe rather involved) cycle of allowed 'simple steps'.

The most convenient tool for studying the effect of cycles is the language of vector fields (Appendix E). The cycle i)-iv) above is just the infinitesimal cycle generated by $H_{\alpha}, H_{\beta}$ (on $P$; its projection to $M$ is generated by $\partial_{\alpha}, \partial_{\beta}$ and the corresponding loop closes exactly since they commute); then the resulting motion (5.3) follows from the formula (see (E.1))

$$
\chi_{-\epsilon}^{H_{\beta}} \circ \chi_{-\epsilon}^{H_{\alpha}} \circ \chi_{\epsilon}^{H_{\beta}} \circ \chi_{\epsilon}^{H_{\alpha}}=\chi_{-\epsilon^{2}}^{\left[H_{\alpha}, H_{\beta}\right]}
$$

and the explicit computation of the needed commutator :

$$
\left[H_{\alpha}, H_{\beta}\right]=R\left\{\sin (\varphi+\beta) \partial_{x}-\cos (\varphi+\beta) \partial_{y}-\frac{1}{l} \cos \beta \partial_{\varphi}\right\}
$$

Since (5.3) is not enough, we can try the 'higher' (iterated) commutators. There are two of them to be computed and the results are :

$$
\left[H_{\alpha},\left[H_{\alpha}, H_{\beta}\right]\right]=\frac{R^{2}}{l}\left(\cos \varphi \partial_{y}-\sin \varphi \partial_{x}\right)
$$




$$
\left[H_{\beta},\left[H_{\alpha}, H_{\beta}\right]\right]=H_{\alpha}-\partial_{\alpha} \equiv \partial_{\alpha}^{h}-\partial_{\alpha}
$$

Both of these results deserve some attention. First, note that the r.h.s of (5.6) just generates the wanted motion (5.1)! In more detail, the identity (see E.3)

$$
\chi_{-\epsilon}^{H_{\beta}} \circ \chi_{-\epsilon}^{H_{\alpha}} \circ \chi_{\epsilon}^{H_{\beta}} \circ \chi_{\epsilon}^{H_{\alpha}} \circ \chi_{-\epsilon^{2}}^{H_{\alpha}} \circ \chi_{-\epsilon}^{H_{\alpha}} \circ \chi_{-\epsilon}^{H_{\beta}} \circ \chi_{\epsilon}^{H_{\alpha}} \circ \chi_{\epsilon}^{H_{\beta}} \circ \chi_{\epsilon^{2}}^{H_{\alpha}}=\chi_{-\epsilon^{4}}^{\left[H_{\alpha},\left[H_{\alpha}, H_{\beta}\right]\right]}
$$

tells us that the iterated cycle standing on the 1.h.s. of (5.8) (try to draw a picture modifying appropriately Fig.4 !) results in

$$
(x, y, \varphi) \mapsto\left(x+\epsilon^{4} \frac{R^{2}}{l} \sin \varphi, y-\epsilon^{4} \frac{R^{2}}{l} \cos \varphi, \varphi\right)
$$

which is just the pure translation perpendicular to the tie rod. Note that this type of motion is very slow and laborious : it is necessary to perform ten 'simple' steps (of the order $\epsilon$ or $\epsilon^{2}$ ) to produce effectively a single step (which is of the order $\epsilon^{4}$ ) in the 'right' direction.

The same treatment applied to (5.7) leads to the identity

$$
\chi_{-\epsilon}^{H_{\beta}} \circ \chi_{-\epsilon}^{H_{\alpha}} \circ \chi_{\epsilon}^{H_{\beta}} \circ \chi_{\epsilon}^{H_{\alpha}} \circ \chi_{-\epsilon^{2}}^{H_{\beta}} \circ \chi_{-\epsilon}^{H_{\alpha}} \circ \chi_{-\epsilon}^{H_{\beta}} \circ \chi_{\epsilon}^{H_{\alpha}} \circ \chi_{\epsilon}^{H_{\beta}} \circ \chi_{\epsilon^{2}}^{H_{\beta}}=\chi_{-\epsilon^{4}}^{\left[H_{\beta},\left[H_{\alpha}, H_{\beta}\right]\right]}
$$

which shows that performing the (iterated) cycle standing on the l.h.s. of (5.10) the car moves just like if the driver simply moved forth, but the front wheel did not rotate (no change of $\alpha$ at all : ideally slipping contact ice on the road).

As mentioned in Appendix E, the possibility of producing 'forbidden' motions by means of the cycles composed of 'allowed' steps leans heavily on the fact that the curvature of the connection in question does not vanish $(\leftrightarrow$ the horizontal lifts of coordinate basis vectors do not commute). The (Lie algebra valued) curvature 2-form, which happens [7] to be the measure of this non-commutation, can be computed easily explicitly (using the formula E.8) here and the result reads

$$
\begin{aligned}
\Omega \equiv \text { hor } d \omega & =\Omega^{a} e_{a}=\Omega^{0} e_{0}+\Omega^{1} e_{1}+\Omega^{2} e_{2}= \\
& =\left(\begin{array}{ccc}
0 & \Omega^{0} & 0 \\
-\Omega^{0} & 0 & 0 \\
\Omega^{1} & \Omega^{2} & 0
\end{array}\right)
\end{aligned}
$$

where

$$
\begin{gathered}
\Omega^{0}=\frac{R}{l} \cos \beta d \alpha \wedge d \beta \\
\Omega^{1}=\left\{-R \sin (\beta+\varphi)+\frac{R}{l} y \cos \beta\right\} d \alpha \wedge d \beta \\
\Omega^{2}=\left\{R \cos (\beta+\varphi)-\frac{R}{l} x \cos \beta\right\} d \alpha \wedge d \beta
\end{gathered}
$$

If one fixes the gauge by choosing the section $\sigma$ (Sec.2), the field strength (in gauge $\sigma$ ) is given as

$$
\begin{aligned}
& \mathcal{F}:=\sigma^{*} \Omega=\mathcal{F}^{a} e_{a}=\mathcal{F}^{0} e_{0}+\mathcal{F}^{1} e_{1}+\mathcal{F}^{2} e_{2}=\left(\begin{array}{ccc}
0 & \mathcal{F}^{0} & 0 \\
-\mathcal{F}^{0} & 0 & 0 \\
\mathcal{F}^{1} & \mathcal{F}^{2} & 0
\end{array}\right)=
\end{aligned}
$$

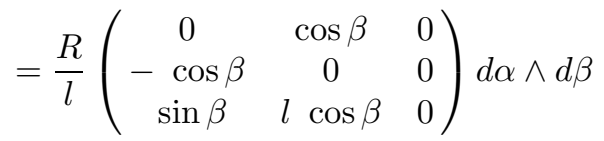

\section{Particle fields}

The gauge potentials $\mathcal{A} \equiv \sigma^{*} \omega$ (and the field strengths $\mathcal{F} \equiv \sigma^{*} \Omega$ ) do not exhaust all the building blocks of the gauge theory of elementary particles - there are also particle fields there : particles interact via gauge fields (bosons). 
In our model of the kinematics of a car we used only the 'connection part of the theory' yet. The question arises whether there is an object here which is described mathematically by a particle field and whether some standard computation with it does make sense in this context.

If $V$ is a vector space in which a representation $\rho$ acts then [7] particle field of type $\rho$ is a $V$-valued function on $P$ which transforms according to the representation $\rho$ with respect to the action of $G$ on $P$; in our model it means

$$
\psi: P \rightarrow V
$$

such that

$$
\psi\left(R_{\mathcal{B}} p\right)=\rho\left(\mathcal{B}^{-1}\right) \psi(p)
$$

Here we give a simple example of such $\psi$. Let $V=\mathbb{R}^{2}$ and define the function $\psi$ on $P$

$$
\psi:(\alpha, \beta, x, y, \varphi) \mapsto\left(\begin{array}{c}
\cos \varphi \\
\sin \varphi
\end{array}\right) .
$$

Then $\psi(p)$ just gives the components of the unit vector $\vec{e}$ fixed on the car and directed along the tie rod (Fig.1). According to Appendix A the action of $E(2)$ has the explicit form

$$
\begin{gathered}
(\alpha, \beta, x, y, \varphi) \mapsto R_{\mathcal{B}}(\alpha, \beta, x, y, \varphi) \equiv \\
\equiv\left(\alpha, \beta, x \cos \Theta-y \sin \Theta+b_{1}, x \sin \Theta+y \cos \Theta+b_{2}, \varphi+\Theta\right)
\end{gathered}
$$

and thus if

$$
\psi(p) \leftrightarrow\left(\begin{array}{c}
\cos \varphi \\
\sin \varphi
\end{array}\right)
$$

then

$$
\begin{gathered}
\psi\left(R_{\mathcal{B}} p\right) \leftrightarrow\left(\begin{array}{c}
\cos (\varphi+\Theta) \\
\sin (\varphi+\Theta)
\end{array}\right)= \\
=\left(\begin{array}{cc}
\cos \Theta & -\sin \Theta \\
\sin \Theta & \cos \Theta
\end{array}\right)\left(\begin{array}{c}
\cos \varphi \\
\sin \varphi
\end{array}\right)=: \rho\left(\mathcal{B}^{-1}\right)\left(\begin{array}{c}
\cos \varphi \\
\sin \varphi
\end{array}\right)
\end{gathered}
$$

where the representation $\rho$ of $E(2)$ in $V \equiv \mathbb{R}^{2}$ is given by

$$
\rho(\mathcal{B}) \equiv \rho((B(\Theta), b))=\left(\begin{array}{cc}
\cos \Theta & \sin \Theta \\
-\sin \Theta & \cos \Theta
\end{array}\right)
$$

Thus our $\psi$ is the particle field of type $\rho$ given by $(6.7)$.

Let the motion in $M$ be given by $m(t) \leftrightarrow(\alpha(t), \beta(t))$. Then the change $\delta \vec{e}$ of the vector $\vec{e}$ between $t$ and $t+\delta t$ can be computed as

$$
\delta \vec{e} \leftrightarrow \delta t \dot{m}^{h} \psi=\frac{\delta t \dot{\alpha} R}{l} \sin \beta\left(\begin{array}{c}
-\sin \varphi \\
\cos \varphi
\end{array}\right)
$$

Since $\left(\begin{array}{c}-\sin \varphi \\ \cos \varphi\end{array}\right)$ is just the unit vector orthogonal to $\vec{e}$, the net angle of rotation of $\vec{e}$ is

$$
\frac{\delta t \dot{\alpha} R}{l} \sin \beta \equiv \frac{\delta \alpha R}{l} \sin \beta
$$

which can be checked by inspection of Fig.1. The same angle can be computed within the gauge fixation $\sigma$, too, making use of the covariant derivative of

$$
\Phi:=\sigma^{*} \psi=\left(\begin{array}{l}
1 \\
0
\end{array}\right) \leftrightarrow \overrightarrow{e_{1}}
$$

viz. (some concepts not mentioned in this paper [7] are needed for it)

$$
\begin{aligned}
\delta \Phi & =\delta t \nabla_{\dot{m}}\left(\sigma^{*} \psi\right)=\delta t<\sigma^{*} D \psi, \dot{m}>= \\
& =\frac{\delta \alpha R}{l} \sin \beta\left(\begin{array}{c}
0 \\
1
\end{array}\right) \leftrightarrow \frac{\delta \alpha R}{l} \sin \beta \overrightarrow{e_{2}}
\end{aligned}
$$


or

$$
\delta \overrightarrow{e_{1}}=\frac{\delta \alpha R}{l} \sin \beta \overrightarrow{e_{2}}
$$

in concord with (6.9).

\section{Conclusions and comments}

In this paper we have presented in some detail a gauge-theoretic approach to the kinematics of a motion of a car. It can serve as still another example of application of the ideas and techniques of the mathematics of gauge fields and related structures within rather mundane context of the elementary (classical) mechanics (as opposed to their standard occurrence in 'noble' = 'fundamental' physics).

The formal scheme is the same here like in [1] or [3] : there is a 'total' configuration space $(P$ here $\leftrightarrow$ the space of located shapes in [1] $\leftrightarrow X$ in [3]) which happens to carry the structure of the the total space of the principal fibre bundle. The group $G$ acts there $(E(2)$ here $\leftrightarrow S O(3)$ in $[1] \leftrightarrow S O(d)$ in [3]) and the space of orbits of this action ( $M$ here $\leftrightarrow$ the space of unlocated shapes in [1] $\leftrightarrow \tilde{X}$ in [3]), the base of the bundle, represents the 'directly controllable part' of the total configuration space. The connection in $\pi: P \rightarrow M$ provides the bridge linking the motions in these two spaces.

The main difference lies in the physical origin of the connection in question : here (and also in [5]) it encodes the constraints expressing the no-slipping (direct) contact of the car with the road whereas in [1],[3] and [4] it results from the conservation laws (of the linear as well as the angular momentum) in 'nothing to push against' situation, i.e. the constraints enter the problem dynamically.

\section{Appendix $\mathrm{A}$ : The action of $\mathrm{E}(2)$ on $\mathcal{E}$ and on $P$}

Let $B \in S O(2), b \equiv\left(b_{1}, b_{2}\right) \in \mathbb{R}^{2}$. Then one can define the transformation of the points $\chi \equiv\left(x_{1}, x_{2}\right) \in \mathbb{R}^{2}$ by the couple $(B, b)$ by

$$
\chi \mapsto \chi B+b=: \mathcal{R}_{(B, b)} \chi
$$

Geometrically it represents the rotation by $\Theta$ around the origin (if $\left.B=\left(\begin{array}{cc}\cos \Theta & \sin \Theta \\ -\sin \Theta & \cos \Theta\end{array}\right)\right)$ followed by the translation by $\left(b_{1}, b_{2}\right)$, i.e. the Euclidean transformation of $\chi$ by $(B, b) \in E(2)$. The rule (A1) can be written in purely matrix form (which is advantageous for manipulations with the gauge potentials) using the following standard trick : let us associate the $3 \times 3$ matrix $\mathcal{B}$ and the row vector $\eta$ with the couple $(B, b)$ and the row vector $\chi$ respectively according to

$$
\mathcal{B}:=\left(\begin{array}{cc}
B & 0 \\
b & 1
\end{array}\right) \quad \eta:=(\chi, 1) \equiv\left(x_{1}, x_{2}, 1\right)
$$

Then the matrix multiplication of $\eta$ by $\mathcal{B}$ gives

$$
\eta \mathcal{B}=\eta^{\prime}=(\chi B+b, 1) \equiv\left(\mathcal{R}_{(B, b)} \chi, 1\right)
$$

i.e. the rule (A1) is reproduced from the matrix multiplication of the auxiliary quantities $\eta$ and $\mathcal{B}$.

The action given by (A1) or (A3) transforms the $\left(x_{1}, x_{2}\right)$-plane 'rigidly', i.e. all distances are preserved $\left(\mathcal{R}_{\mathcal{B}} \equiv \mathcal{R}_{(B, b)}\right.$ is an isometry). It enables then to define the action $\hat{R}_{\mathcal{B}}$ of $\mathrm{E}(2)$ on the space $\mathcal{E}$ of the locations of the tie rod, transforming simply both endpoints by $\mathcal{R}_{\mathcal{B}}$. If the coordinates $(x, y, \varphi)$ are introduced to $\mathcal{E}$ according to the Fig.1, one obtains

$$
(x, y, \varphi) \mapsto\left(x \cos \Theta-y \sin \Theta+b_{1}, x \sin \Theta+y \cos \Theta+b_{2}, \varphi+\Theta\right) \equiv \hat{R}_{\mathcal{B}}(x, y, \varphi)
$$

Notice that the general position $(x, y, \varphi)$ of the rod can be reached from the reference one $(0,0,0)$ (the rod being situated on the $x_{1}$-axis left to the origin) by means of the unique $\hat{R}_{\mathcal{B}}$ :

$$
\hat{R}_{\mathcal{B}}(0,0,0)=(x, y, \varphi) \quad \text { for } \quad B=\left(\begin{array}{cc}
\cos \varphi & \sin \varphi \\
-\sin \varphi & \cos \varphi
\end{array}\right), b=(x, y)
$$


This means that the action $\hat{R}_{\mathcal{B}}$ is transitive and free and thus $\mathcal{E}$ is the 'principal E(2)-space'. Note that (A5) gives the diffeomorphism of $\mathcal{E}$ and the group $\mathrm{E}(2)$ itself , too.

Finally the action $R_{\mathcal{B}}$ on $P=M \times \mathcal{E}$ is given by

$$
(m, e) \mapsto\left(m, \hat{R}_{\mathcal{B}} e\right)=: R_{\mathcal{B}}(m, e)
$$

or in coordinates

$$
\begin{gathered}
(\alpha, \beta, x, y, \varphi) \mapsto R_{\mathcal{B}}(\alpha, \beta, x, y, \varphi) \equiv \\
\equiv\left(\alpha, \beta, x \cos \Theta-y \sin \Theta+b_{1}, x \sin \Theta+y \cos \Theta+b_{2}, \varphi+\Theta\right)
\end{gathered}
$$

\section{Appendix B : The Lie algebra $e(2)$ of the group $E(2)$}

According to Appendix A the group $E(2)$ can be realized by the matrices $\mathcal{B}=\left(\begin{array}{cc}B & 0 \\ b & 1\end{array}\right)$, where $B \in S O(2)$. By definition, the Lie algebra $e(2)$ consists then of all $3 \times 3$ matrices $\mathcal{C}$ such that $1+\epsilon \mathcal{C} \equiv \mathcal{B}(\epsilon) \in E(2)$ when the 2-nd order terms in $\epsilon$ are neglected. This leads to $\mathcal{C}=\left(\begin{array}{ll}C & 0 \\ c & 0\end{array}\right)$ with the additional restriction (comming from $\left.B^{T} B=1\right) C^{T}=-C$, or explicitly

$$
\mathcal{C}=\left(\begin{array}{ccc}
0 & \lambda_{0} & 0 \\
-\lambda_{0} & 0 & 0 \\
\lambda_{1} & \lambda_{2} & 0
\end{array}\right) \quad \lambda_{0}, \lambda_{1}, \lambda_{2} \in \mathbb{R}
$$

The matrices

$$
e_{0}=\left(\begin{array}{ccc}
0 & 1 & 0 \\
-1 & 0 & 0 \\
0 & 0 & 0
\end{array}\right) \quad e_{1}=\left(\begin{array}{ccc}
0 & 0 & 0 \\
0 & 0 & 0 \\
1 & 0 & 0
\end{array}\right) \quad e_{2}=\left(\begin{array}{ccc}
0 & 0 & 0 \\
0 & 0 & 0 \\
0 & 1 & 0
\end{array}\right)
$$

can serve then as the basis of $e(2)$; their commutation relations read

$$
\begin{gathered}
{\left[e_{0}, e_{1}\right]=-e_{2}} \\
{\left[e_{0}, e_{2}\right]=+e_{1}} \\
{\left[e_{1}, e_{2}\right]=0}
\end{gathered}
$$

and so the only non-zero structure constants are

$$
c_{10}^{2}=-c_{01}^{2}=c_{02}^{1}=-c_{20}^{1}=1
$$

\section{Appendix C : A computation of the connection form $\omega$}

In general a connection form can be written as follows

$$
\omega=\mathcal{B}^{-1} \bar{\omega} \mathcal{B}+\mathcal{B}^{-1} d \mathcal{B}
$$

where $\bar{\omega} \equiv \bar{\omega}^{a} e_{a}$ is some (yet unknown) $e(2)$-valued 1-form on $M$ and

$$
\mathcal{B}=\left(\begin{array}{ccc}
\cos \varphi & \sin \varphi & 0 \\
-\sin \varphi & \cos \varphi & 0 \\
x & y & 1
\end{array}\right) \quad \in E(2)
$$


The form $\omega$ defines the horizontal directions (the relations between $d \alpha, d \beta, d x, d y$, and $d \varphi$ as a result of the no-slipping contact of the wheels with the road) by the equations $\omega^{a}=0, a=0,1,2$. In particular at the points of the section $\sigma(M) \subset P$, corresponding to the 'standard' position of a car (i.e. for $x=y=\varphi=0$; cf.Sec.2) one has $\mathcal{B}=1=\mathcal{B}^{-1}$ and

$$
\omega_{\mathcal{B}=1}=\bar{\omega}+(d \mathcal{B})_{\mathcal{B}=1}
$$

Thus

$$
\begin{aligned}
\omega_{\mathcal{B}=1} & =\left(\begin{array}{ccc}
0 & \bar{\omega}^{0} & 0 \\
-\bar{\omega}^{0} & 0 & 0 \\
\bar{\omega}^{1} & \bar{\omega}^{2} & 0
\end{array}\right)+\left(\begin{array}{ccc}
0 & d \varphi & 0 \\
-d \varphi & 0 & 0 \\
d x & d y & 0
\end{array}\right)= \\
& =\left(d \varphi+\bar{\omega}^{0}\right) e_{0}+\left(d x+\bar{\omega}^{1}\right) e_{1}+\left(d y+\bar{\omega}^{2}\right) e_{2}
\end{aligned}
$$

The equation $\omega_{\mathcal{B}=1}=0$ by definition singles out the horizontal directions for $x=y=\varphi=0$; it reads

$$
\begin{aligned}
d \varphi & =-\bar{\omega}^{0} \\
d x & =-\bar{\omega}^{1} \\
d y & =-\bar{\omega}^{2}
\end{aligned}
$$

On the other hand the no-slipping contact constraints for the standard position $x=y=\varphi=0$ can be easily read out from the Fig.3 : if $\alpha \mapsto \alpha+\delta \alpha(\delta \alpha \ll 1)$, then $(x, y) \equiv(0,0) \mapsto(\delta \alpha R \cos \beta, \delta \alpha R \sin \beta) \equiv(\delta x, \delta y)$, $(-l, 0) \mapsto(-l+\delta \alpha R \cos \beta, 0) \Rightarrow \varphi \mapsto \varphi+\delta \alpha \frac{R}{l} \sin \beta$; if $\beta \mapsto \beta+\delta \beta(\delta \beta \ll 1)$, then $(x, y, \varphi) \mapsto(x, y, \varphi)$. Thus

$$
\begin{aligned}
& d \varphi=\frac{R}{l} \sin \beta d \alpha \\
& d x=R \cos \beta d \alpha \\
& d y=R \sin \beta d \alpha
\end{aligned}
$$

A comparison with (C5) gives

$$
\begin{gathered}
\bar{\omega}^{0}=-\frac{R}{l} \sin \beta d \alpha \\
\bar{\omega}^{1}=-R \cos \beta d \alpha \\
\bar{\omega}^{2}=-R \sin \beta d \alpha \\
\bar{\omega} \equiv \bar{\omega}^{a} e_{a}=-\frac{R}{l}\left(\begin{array}{ccc}
0 & \sin \beta & 0 \\
-\sin \beta & 0 & 0 \\
l \cos \beta & l \sin \beta & 0
\end{array}\right) d \alpha
\end{gathered}
$$

Inserting this into $(\mathrm{C} 1)$ leads finally to

$$
\omega=\omega^{0} e_{0}+\omega^{1} e_{1}+\omega^{2} e_{2}
$$

where

$$
\begin{gathered}
\omega^{0}=d \varphi-\frac{R}{l} \sin \beta d \alpha \\
\omega^{1}=d x+y \omega^{0}-R \cos (\beta+\varphi) d \alpha \\
\omega^{2}=d y-x \omega^{0}-R \sin (\beta+\varphi) d \alpha
\end{gathered}
$$

Thus the differential constraints in general configuration are $\left(\omega^{a}=0\right)$

$$
\begin{gathered}
d \varphi=\frac{R}{l} \sin \beta d \alpha \\
d x=R \cos (\beta+\varphi) d \alpha \\
d y=R \sin (\beta+\varphi) d \alpha
\end{gathered}
$$

Note the absence of the differential $d \beta$ on the r.h.s. - it reflects the evident fact that turning the steering wheel alone results in no motion of the tie rod. 


\section{Appendix D : Motion of the car with fixed steering wheel}

In the case of a fixed steering wheel $\left(\beta(t)=\beta_{0}=\right.$ const) the parallel transport equations (4.1) read

$$
\begin{gathered}
\varphi^{\prime}(\alpha)=\frac{R}{l} \sin \beta_{0} \\
x^{\prime}(\alpha)=R \cos \left(\beta_{0}+\varphi(\alpha)\right) \\
y^{\prime}(\alpha)=R \sin \left(\beta_{0}+\varphi(\alpha)\right)
\end{gathered}
$$

$\left(\varphi^{\prime}(\alpha) \equiv \frac{d \varphi}{d \alpha}, \ldots\right)$. They are easily solved. If $\beta_{0} \neq 0$, then

$$
\begin{gathered}
\varphi(\alpha)=\varphi_{0}+\alpha \frac{R}{l} \sin \beta_{0} \\
x(\alpha)=x_{0}+\frac{l}{\sin \beta_{0}}\left(\sin \left(\varphi(\alpha)+\beta_{0}\right)-\sin \left(\varphi_{0}+\beta_{0}\right)\right) \\
y(\alpha)=y_{0}-\frac{l}{\sin \beta_{0}}\left(\cos \left(\varphi(\alpha)+\beta_{0}\right)-\cos \left(\varphi_{0}+\beta_{0}\right)\right)
\end{gathered}
$$

and consequently

$$
\left(x(\alpha)-x_{c}\right)^{2}+\left(y(\alpha)-y_{c}\right)^{2}=r_{c}^{2}
$$

where

$$
\begin{gathered}
r_{c} \equiv \frac{l}{\sin \beta_{0}} \\
x_{c} \equiv x_{0}-r_{c} \sin \left(\varphi_{0}+\beta_{0}\right) \\
y_{c} \equiv x_{0}+r_{c} \cos \left(\varphi_{0}+\beta_{0}\right)
\end{gathered}
$$

Thus, as expected, the front wheel draws a circle with the radius $r_{c}$ and the center $\left(x_{c}, y_{c}\right)$. If $\beta_{0}=0$, the equations (D.1) give

$$
\begin{gathered}
\varphi(\alpha)=\varphi_{0} \\
x(\alpha)=x_{0}+\alpha R \cos \varphi_{0} \\
y(\alpha)=y_{0}+\alpha R \sin \varphi_{0}
\end{gathered}
$$

which is a straight line in the direction of the tie rod.

\section{Appendix E : Commutators, infinitesimal cycles and the curvature}

Let $U, V$ be two vector fields on a manifold $\mathcal{M},[U, V]$ their commutator (Lie bracket) and $\chi_{t}^{U}, \chi_{t}^{V}$ and $\chi_{t}^{[U, V]}$ the corresponding flows ( $\chi_{t}^{U}$ is the map $\mathcal{M} \rightarrow \mathcal{M}$ sending each point $x \in \mathcal{M}$ a (parameter) distance $t$ along the integral curve of $U$; it holds $\left.\chi_{(t+s)}^{U}=\chi_{t}^{U} \circ \chi_{s}^{U}=\chi_{s}^{U} \circ \chi_{t}^{U}\right)$. Then a computation shows that up to the second order terms in $\epsilon \ll 1$ the following important identity is valid :

$$
\chi_{-\epsilon}^{V} \circ \chi_{-\epsilon}^{U} \circ \chi_{\epsilon}^{V} \circ \chi_{\epsilon}^{U}=\chi_{-\epsilon^{2}}^{[U, V]}
$$

or equivalently

$$
\chi_{\epsilon^{2}}^{[U, V]} \circ \chi_{-\epsilon}^{V} \circ \chi_{-\epsilon}^{U} \circ \chi_{\epsilon}^{V} \circ \chi_{\epsilon}^{U}=\text { identity on } \mathcal{M}
$$

From these formulae one deduces the standard interpretation of the commutator of two vector fields : the infinitesimal cycle generated by $U$ and $V$ (l.h.s. of (E.1)) does not end at the original point within the accuracy $\epsilon^{2}$ (although it does within the accuracy $\epsilon$ ) but rather one has to add one order smaller step along $[U, V]$ to 
close the loop (l.h.s. of (E.2)).

Now if $V$ is itself a commutator, $V=[W, Z]$, the twofold use of (E.1) yields

$$
\chi_{-\epsilon}^{Z} \circ \chi_{-\epsilon}^{W} \circ \chi_{\epsilon}^{Z} \circ \chi_{\epsilon}^{W} \circ \chi_{-\epsilon^{2}}^{U} \circ \chi_{-\epsilon}^{W} \circ \chi_{-\epsilon}^{Z} \circ \chi_{\epsilon}^{W} \circ \chi_{\epsilon}^{Z} \circ \chi_{\epsilon^{2}}^{U}=\chi_{-\epsilon^{4}}^{[U,[W, Z]]}
$$

Thus the computation of 'simple' $([U, V])$ and iterated $([U,[W, Z]])$ commutators tells us what is the result of a simple (4 steps) and iterated (4 steps, but two of them being themselves results of 4 steps, i.e. together 10 simple steps) cycles respectively (the higher iterated commutators can be treated in the same way).

All said until now is valid for any vector fields on any manifold. In the case when the vector fields in question are horizontal lifts, the resulting commutator can be expressed in terms of the curvature of the connection. For doing this we need first the concept of the fundamental fields of the action $R_{\mathcal{B}}$. By definition the field $\xi_{\mathcal{C}}, \mathcal{C} \equiv \mathcal{C}^{a} e_{a} \in e(2)$, generates the motion of any point $p$ under the action of the one-parameter subgroup $\mathcal{B}(\lambda)=e^{\lambda \mathcal{C}}$, i.e. for $p(\lambda):=R_{\mathcal{B}(\lambda)} p$

$$
\xi_{\mathcal{C}}(p):=\dot{p}(0)
$$

For the basis elements $e_{0}, e_{1}, e_{2} \in e(2)$ we obtain explicitly

$$
\begin{gathered}
\xi_{0} \equiv \xi_{e_{0}}=-y \partial_{x}+x \partial_{y}+\partial_{\varphi} \\
\xi_{1} \equiv \xi_{e_{1}}=\partial_{x} \\
\xi_{2} \equiv \xi_{e_{2}}=\partial_{y}
\end{gathered}
$$

and in general

$$
\xi_{\mathcal{C}} \equiv \xi_{\mathcal{C}^{a} e_{a}}=\mathcal{C}^{a} \xi_{e_{a}} \equiv \mathcal{C}^{a} \xi_{a}
$$

These fields are purely vertical (directed along the fiber), since (by definition) the action is vertical ( $p$ and $R_{\mathcal{B}} p$ lie in the same fiber for all $p, \mathcal{B})$. Now the relevant formula for the commutator of $H_{\alpha}$ and $H_{\beta}$ is

$$
\left[H_{\alpha}, H_{\beta}\right]=\xi_{-\Omega\left(H_{\alpha}, H_{\beta}\right)}=-\Omega^{a}\left(H_{\alpha}, H_{\beta}\right) \xi_{a}
$$

where the (e(2)-valued ) curvature 2-form $\Omega=\Omega^{a} e_{a}$ is given by

$$
\Omega^{a}=d \omega^{a}+\frac{1}{2} c_{b c}^{a} \omega^{b} \wedge \omega^{c}
$$

( $c_{b c}^{a}$ being the structure constants; they are computed in Appendix B). The formula (E.7) shows that

i) $\left[H_{\alpha}, H_{\beta}\right]$ is non-zero if and only if $\Omega$ is non-zero

ii) $\left[H_{\alpha}, H_{\beta}\right]$ is purely vertical $\Rightarrow$ the corresponding cycle generates 'forbidden' motion.

The explicit form of $\Omega$ in our case is displayed in Sec.5 (see (5.11), (5.12)).

\section{References}

a) Present address : Department of Theoretical Physics, Comenius University, Mlynská dolina F2, 84215 Bratislava, Slovakia; e-mail: fecko@fmph.uniba.sk (published in Il Nuovo Cimento B, Vol 111 (11) 1315-1332 (1996))

[1] A.Shapere, F.Wilczek: "Gauge kinematics of deformable bodies", Am.J.Phys.57 (6),514-518, June 1989

[2] A.Shapere, F.Wilczek: "Geometry of Self-Propulsion at Low Reynolds number, J.Fluid.Mech. 198, 557-585 (1989)

[3] A.Guichardet: "On rotation and vibration motions of molecules", Ann.Inst.Henri Poincar, Vol.40, n.3, 1984, p.329-342

[4] M.Fecko : "Falling cat" connections and the momentum map, J.Math.Phys. 36 (12) 6709-6719 (1995) (available also as physics/9702010 at http://xxx.lanl.gov )

[5] M.Fecko : U(1)-gauge structure associated with a motion of a guitar string, Acta Physica Slovaca vol.44, No.6, 445-449 (1994)

[6] E.Nelson : Tensor analysis, Princeton Univ. Press 1967, p.33-36

[7] A.Trautman : Differential geometry for physicists, Bibliopolis, Napoli, 1984, p.88-89,102-103 